\title{
Transcriptional fingerprints of antigen-presenting cell subsets in the human vaginal mucosa and skin reflect tissue-specific immune microenvironments
}

\author{
Dorothée Duluc ${ }^{1 \dagger}$, Romain Banchereau ${ }^{1 \dagger}$, Julien Gannevat ${ }^{1}, L^{\prime}$ uann Thompson-Snipes ${ }^{1}$, Jean-Philippe Blanck ${ }^{1}$ \\ Sandra Zurawski ${ }^{1}$, Gerard Zurawski ${ }^{1}$, Seunghee Hong ${ }^{1}$, Jose Rossello-Urgell ${ }^{1}$, Virginia Pascual ${ }^{1}$, Nicole Baldwin ${ }^{1}$, \\ Jack Stecher ${ }^{2}$, Michael Carley ${ }^{2}$, Muriel Boreham ${ }^{2}$ and SangKon Oh ${ }^{1 *}$
}

\begin{abstract}
Background: Dendritic cells localize throughout the body, where they can sense and capture invading pathogens to induce protective immunity. Hence, harnessing the biology of tissue-resident dendritic cells is fundamental for the rational design of vaccines against pathogens.

Methods: Herein, we characterized the transcriptomes of four antigen-presenting cell subsets from the human vagina (Langerhans cells, CD14 and CD14 ${ }^{+}$dendritic cells, macrophages) by microarray, at both the transcript and network level, and compared them to those of three skin dendritic cell subsets and blood myeloid dendritic cells.

Results: We found that genomic fingerprints of antigen-presenting cells are significantly influenced by the tissue of origin as well as by individual subsets. Nonetheless, CD14 populations from both vagina and skin are geared towards innate immunity and pro-inflammatory responses, whereas CD14 populations, particularly skin and vaginal Langerhans cells, and vaginal CD14- dendritic cells, display both Th2-inducing and regulatory phenotypes. We also identified new phenotypic and functional biomarkers of vaginal antigen-presenting cell subsets.

Conclusions: We provide a transcriptional database of 87 microarray samples spanning eight antigen-presenting cell populations in the human vagina, skin and blood. Altogether, these data provide molecular information that will further help characterize human tissue antigen-presenting cell lineages and their functions. Data from this study can guide the design of mucosal vaccines against sexually transmitted pathogens.
\end{abstract}

\section{Background}

Dendritic cells (DCs) are professional antigen-presenting cells (APCs) that can induce and direct host immune responses towards immunity or tolerance [1]. DCs disseminate throughout the body, sensing invading pathogens in various tissues, including the skin [2-4] and mucosa [5-7]. Therefore, defining the biology of tissue-resident DCs is fundamental for the understanding of tissue-specific immune microenvironments and for the rational design of

\footnotetext{
*Correspondence: sangkono@baylorhealth.edu

${ }^{\dagger}$ Equal contributors

'Baylor Institute for Immunology Research, 3434 Live Oak St, Dallas, TX 75204, USA

Full list of author information is available at the end of the article
}

vaccines that can mount protective immunity in these tissues.

Sexually transmitted microbial pathogens, including viruses and bacteria [8,9], are a major public health burden worldwide. The human vaginal mucosa is the main entry site of these pathogens and therefore has long been attractive as a potential site for mounting protective mucosal immunity. However, the vaginal mucosa, a site constantly exposed to foreign antigens, is also thought to be a unique tolerogenic microenvironment that tightly regulates unwanted immune responses [10-12]. Nonetheless, the immunology of the human vagina remains poorly understood.

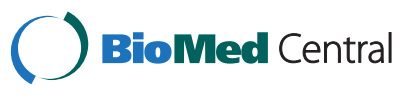


We recently reported the presence of four major subsets of APCs in the human vaginal mucosa, including Langerhans cells (LCs) in the epithelium, and CD14 DCs, CD $14^{+}$DCs and macrophages (Møs) in the lamina propria (LP) $[5,6]$. These mucosal APC subsets display common and unique functions in directing T-cell responses in vitro [5,6], as do subsets of DCs isolated from human skin [2-4]. Importantly, DCs can display functional specialization and plasticity in response to external and internal stimuli [13,14], which can determine the outcome of host immune responses. Recent evidence further indicates that these characteristics of DCs can be influenced by tissue-specific physical and biological factors $[15,16]$. One can thus hypothesize that the same DC subsets localized in different tissues could display distinct functions in response to the same antigens. These differences may also influence the type of immunity established in different human anatomical sites. As such, vaccines delivered to skin DCs can elicit systemic immunity but are not sufficient to mount mucosal immunity $[6,10,17]$.

Systems biology approaches provide snapshots of genetic, transcriptional and protein networks, enabling the phenotypic and functional analysis of the immune system [18-20]. In this study, we investigated the phenotype and function of human vaginal DC subsets by microarray transcriptional profiling and compared them with those of DCs from human skin and blood. This study provides fundamental information for the immunology of human vaginal mucosa versus skin, which can eventually guide the rational design of efficacious vaccines against sexually transmitted pathogens.

\section{Methods}

\section{Samples}

Vaginal and skin tissues were obtained from female patients who underwent pelvic or cosmetic surgeries under protocols approved by the Institutional Review Board of Baylor Research Institute (Dallas, TX, USA). Informed consent was waived by the institutional review board (IRB 008-227) for tissue samples. This study conforms to the Helsinki Declaration. Patients were not infected with HIV, hepatitis $C$ virus or tuberculosis and did not display inflammation in the tissues. Written informed consent was obtained from healthy female volunteers to use their blood in this study, and the protocol was approved by the IRB (IRB 012-200) of Baylor Research Institute.

\section{Vaginal and skin antigen-presenting cell isolation}

Tissue biopsies were cut into $1 \mathrm{~cm}^{2}$ pieces and incubated in phosphate-buffered saline containing bacterial protease dispase type 2 (Roche Applied Science, Indianapolis, IN, USA) and antibiotic/antimycotic solution (Invitrogen, Carlsbad, CA, USA) overnight at $4^{\circ} \mathrm{C}$. Epithelium and LP were then separated. The LP was cut into smaller pieces
(1 to $5 \mathrm{~mm}^{2}$ ). Epithelial sheets and LP pieces were incubated at $37^{\circ} \mathrm{C}$ in RPMI 1640 (Invitrogen) supplemented with HEPES buffer (Invitrogen), antibiotic/antimycotic (Invitrogen), L-glutamine, nonessential amino acids, sodium pyruvate (Sigma Aldrich, St. Louis, MO, USA) and $10 \%$ fetal calf serum (HyClone, Logan, UT, USA). After 2 days, the cells that migrated into the medium were further enriched by Ficoll-sodium diatrizoate gradient (Lymphocyte Separation Medium, MP Biomedicals, Solon, $\mathrm{OH}, \mathrm{USA}$ ). Cells were stained with 7-AAD (Biolegend, San Diego, CA, USA), anti-HLA-DR-AF700 (Biolegend), antiLangerin-PE (Beckman Coulter, Brea, CA, USA), antiCD1c-FITC (Invitrogen) and CD14-eFluor450 (eBioscience, San Diego, CA, USA). HLA-DR ${ }^{+}$cells were gated and Langerin $^{+} \mathrm{LCs}, \mathrm{CD} 1 \mathrm{c}^{+} \mathrm{CD} 14^{-} \mathrm{DCs}, \mathrm{CD} 1 \mathrm{c}^{+} \mathrm{CD} 14^{+} \mathrm{DCs}$ and CD1c ${ }^{-}$CD $14^{+}$Møs were sorted by FACS Aria II (BD Biosciences, San Jose, CA, USA) (Figure 1a, b). To purify HLA-DR cells, single cell suspensions of epithelium and LP were mixed and subsequently sorted (Figure 1b). Skin biopsies were processed similarly. Langerin ${ }^{+}$cells from the epidermis (sLCs; note that cell types prefixed by 's' refer to skin cells) as well as $\mathrm{CD} 1 \mathrm{c}^{+} \mathrm{CD} 14^{-} \mathrm{DCs}$ and $\mathrm{CD} 1 \mathrm{c}^{+} \mathrm{CD} 14^{+}$ DCs cells from the dermis were sorted by FACS Aria II (BD Biosciences). As previously described [3], sCD14- DCs were $\mathrm{CD}^{+}{ }^{+}$, while $\mathrm{sCD} 14^{+} \mathrm{DCs}$ were $\mathrm{CD} 1 \mathrm{a}^{-}$and sLCs were $\mathrm{CD} 1 \mathrm{a}^{\text {high }}$ (Additional file 1).

\section{Blood dendritic cell isolation}

Peripheral blood mononuclear cells from healthy volunteers were isolated by density gradient centrifugation using FicollPaque $^{\text {Th }}$ PLUS (GE Healthcare, Stockholm, Sweden). Blood DCs were enriched from these cells using a Stemcell PanDC kit (Stemcell, Vancouver, BC, Canada) according to the manufacturer's protocol. Cells were then stained with LinFITC (BD Biosciences), CD123-PE (Biolegend), CD11c-APC (BD Biosciences) and HLA-DR-Pacific Orange (Invitrogen). $\mathrm{mDC}\left(\mathrm{Lin}^{-} \mathrm{HLA}-\mathrm{DR}^{+} \mathrm{CD} 11 \mathrm{c}^{+} \mathrm{CD} 123^{-}\right)$were then sorted by FACS Aria (BD Biosciences) (Figure 1a, b).

\section{Immunofluorescence and microscopy}

Cryo-sections were fixed in cold acetone, dried and blocked for non-specific fluorescence with Fc Receptor Block and Background Buster (Innovex Biosciences, Richmond, CA, USA). Sections were stained with the indicated antibodies and subsequently stained with DAPI (Invitrogen). Digital images were taken using an Olympus BX51 with a Planapo20/0.7 or Planapo40/0.95 objective, a Roper Coolsnap HQ camera and Metamorph software (Molecular Devices, Sunnyvale, CA, USA). Images were acquired using the same exposures for antibody and isotype staining and identical scaling was applied. 


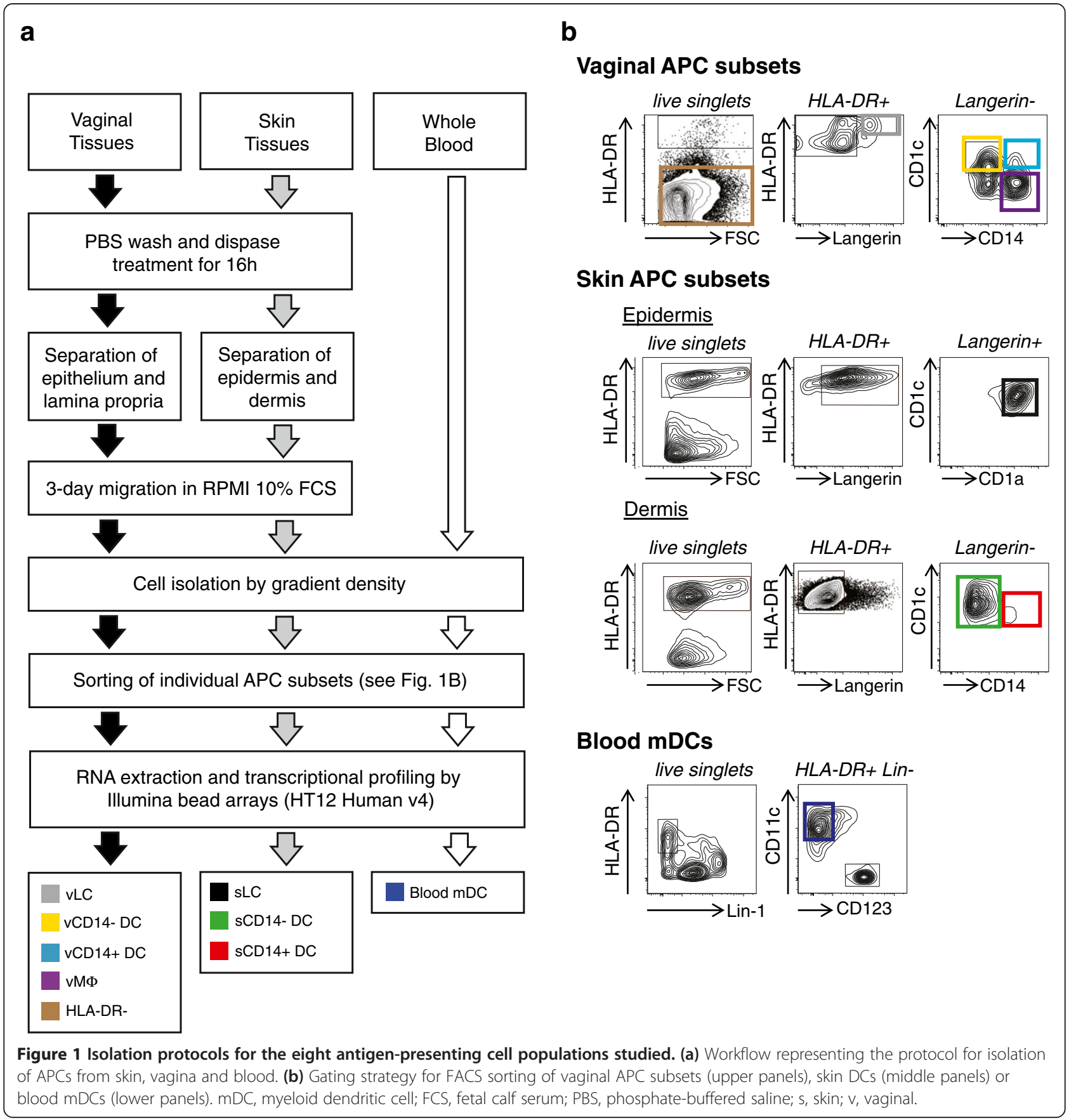

\section{Cell phenotype}

Cells were stained with 7-AAD, anti-HLA-DR-AF700, anti-Langerin PE or anti-Langerin AF488 (in-house), antiCD1c-AF647 (Biolegend) and anti-CD14-eFluor450. Cells were also stained with anti-LOX-1 (clone 15C4, in-house) [21], anti-DC-SIGN (BD Biosciences), anti-DC-ASGPR, [21] anti-DCIR (clone 9E8, in-house), anti-DEC205 (Biolegend) and anti-CD40 (BD Biosciences). Phenotypes of vaginal APCs were analyzed by flow cytometry on an LSR II (BD Biosciences).
mRNA preparation and hybridization

Total RNA was isolated from cell lysates using the ArrayPure-Nano-scale RNA Purification Kit (Epicentre, Madison, WI, USA) according to the manufacturer's instructions. RNA (250 ng) from all samples passing quality control was amplified and labeled using the TargetAmp $^{\text {Tx }}$ 2-Round aRNA Amplification Kit 2.0 (Epicentre). Amplified labeled RNA (750 ng) was hybridized overnight to Illumina HT12 V4 beadchips (Illumina, San Diego, CA, USA). Chips were scanned on an 
Illumina BeadStation 500 following the manufacturer's protocols.

\section{Data pre-processing and batch correction}

Raw data were normalized (average) in Genome Studio ${ }^{\text {tm }}$ (Illumina). Data were normalized to the median of the 80 samples in Genespring 7.3 (Agilent Technologies, Santa Clara, CA, USA). To identify technical sources of variability, we conducted principal component analysis (PCA) and principal variance component analysis (PVCA) using the 27,935 detected genes (Illumina detection $P$-values $<0.01$ in at least 1 of 80 samples). To correct the batch effect, we conducted Combat correction using the SVA package from R/Bioconductor [22]. The batch effect's contribution to variability was removed, as shown by PVCA in Additional file 2.

\section{Analysis of variance}

One-way Welch analysis of variance (ANOVA) was conducted using a $P$-value cutoff of 0.05 and BenjaminiHochberg multiple testing correction.

\section{Principal variance component analysis}

The weighted average proportion variance was calculated with the R/Bioconductor package 'pvca' (version 1.0.0) [23]. The threshold used for the minimum amount of the variance explained by the selected principal components was 0.5 .

\section{Results}

\section{Eight populations of human antigen-presenting cells}

We isolated eight populations of human APCs - four vaginal populations, three skin populations and blood $\mathrm{mDCs}$ - and characterized their transcriptional profiles by microarray. The isolation protocol and sort gating strategy are presented in Figure 1. The same protocol was used to isolate cells from the vaginal mucosa and the skin: i) the biopsy samples were cultured overnight in the presence of dispase; ii) the skin epidermis and the dermis, or vaginal epithelium and LP, were separated and further incubated to allow APC migration into the media (Figure 1a); and iii) the cells were sorted using the same antibodies (Figure 1b).

From the vaginal epithelium and LP, vaginal vLCs, vCD14 DCs, vCD14 ${ }^{+}$DCs and vMøs were obtained. HLA-DR ${ }^{-}$cells were controls. From the skin, epidermal sLCs, sCD14- DCs and sCD14 ${ }^{+}$DCs were obtained. Blood $\mathrm{mDC}$ were sorted from buffy coats.

\section{Global transcriptional relationships across APC populations and tissues}

To compare the transcriptional profiles of these eight APC populations, we first conducted correlation analysis of all samples starting from the 27,935 transcripts detected in this dataset (Illumina detection $P$-values $<0.01$ in at least 1 of 80 samples). The matrix in Figure 2a displays correlations between samples within and across cell populations and tissues. sLCs and mDCs showed patterns distinct from all other populations. Dermal sCD14 and $\mathrm{sCD}^{-} 4^{+}$DCs displayed high correlation, highlighting their transcriptional similarities. Although the four vaginal APC subsets displayed significant variability within each population, vLCs correlated more strongly with vCD14- DCs, while vCD $14^{+}$DCs correlated more strongly with vMøs. This observation is in agreement with the previously described functional similarities between these two pairs of human vaginal APC subsets [5].

One-way ANOVA identified 9,955 differentially expressed transcripts (DETs) between the eight APC populations. These transcripts were hierarchically clustered and represented as a heatmap in Figure 2b. To identify the known experimental parameters that most influenced sample clustering, we conducted PVCA. Taking into account tissue, cell population, and the interaction between these two parameters, we found that the tissue of origin (skin, vagina or blood) explained the largest proportion of variance (0.37), followed by cell population (0.17) (Figure 2c). This observation was further substantiated using PCA (Figure 2d) and hierarchical clustering (Additional file 3), where samples primarily clustered by tissue. In the vagina, vLCs and vCD14DCs clustered together, while $\mathrm{vCD}^{+} 4^{+} \mathrm{DCs}$ and vMøs formed another cluster, consistent with the correlation levels observed between these APC subsets in Figure 2a.

Finally, Tukey's test following ANOVA identified the transcripts differentially regulated between populations in a pair-wise fashion. The results are displayed as a heatmap representing the number of DETs between each population pair (Figure 2e). The most distant populations were vMøs and sLCs (694 DETs), while vLCs and vCD14 DCs were the most similar (only 5 DETs).

Thus, unsupervised analysis identified relative transcriptional similarities and differences between the eight APC populations considered, highlighting the importance of the tissue of origin in determining global transcriptional fingerprints.

\section{Tissue- and population-specific transcriptional profiles}

To further understand the differences in transcriptional profiles between these eight APC populations, we conducted additional variance analysis. The 9,955 DETs resulting from the ANOVA (Figure $2 \mathrm{~b}$ ) were filtered based on the $P$-value for each population. For each subset, we selected transcripts that were differentially expressed from the mean of all samples $(P<0.05)$, requiring that the $P$-value for all populations other than the one of interest remains greater than 0.05 (Figure 3a). This approach also enables the identification of genes specifically modulated in a group of populations 


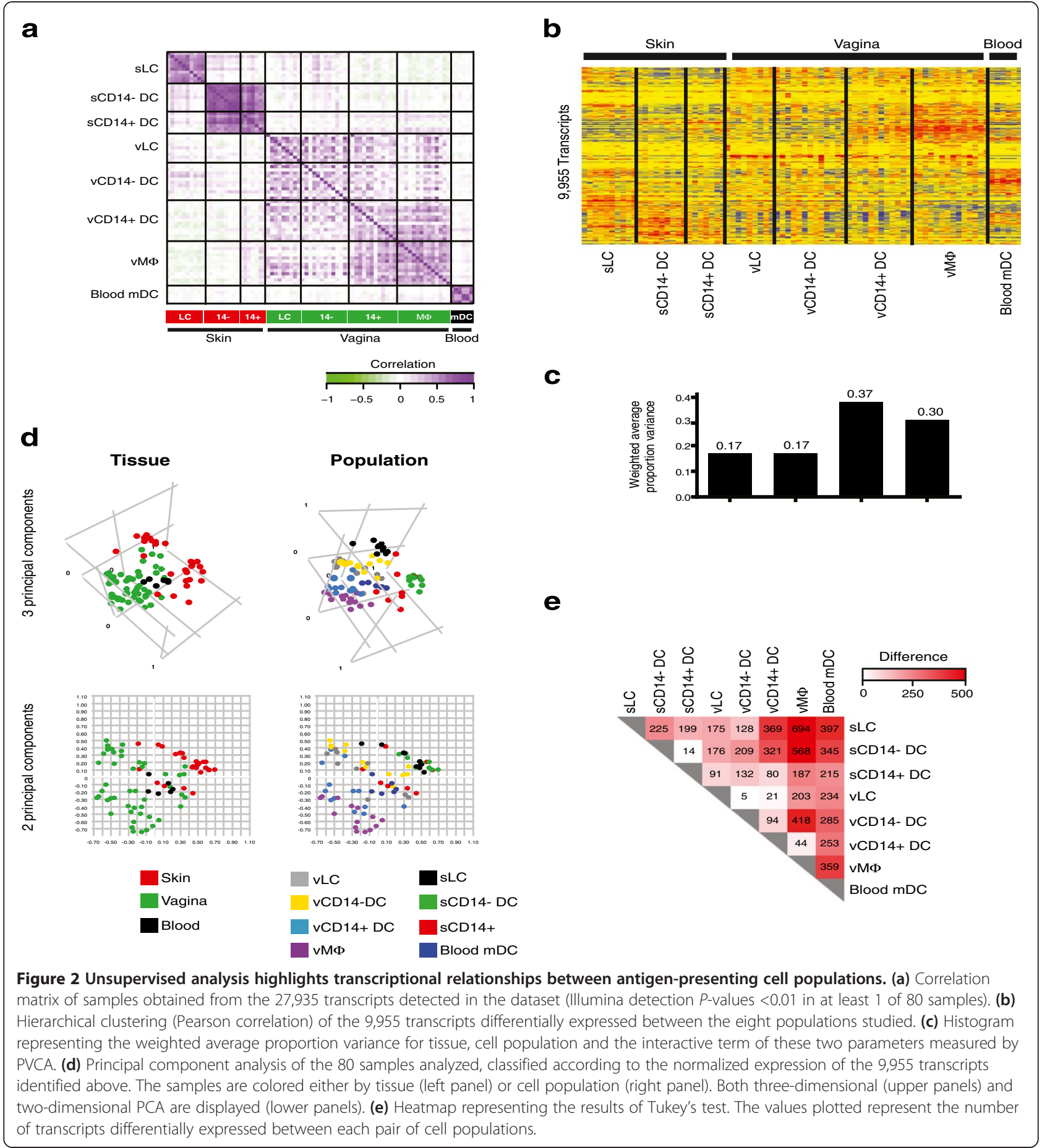

compared with others, such as genes specific for skin $(P<$ 0.05 in sLCs, sCD14 $4^{-}$DCs and sCD14 $\left.{ }^{+} \mathrm{DCs}\right)$, vagina $(P<$ 0.05 in vLCs, vCD14- DCs, vCD14 $4^{+}$DCs and vMøs) or LCs $(P<0.05$ in sLCs and vLCs), for example. The results of this analysis are displayed in Figure 3b, with the $P$-value for each population displayed on the right panel of the heatmap. To focus on over-expressed transcripts, we additionally filtered significant genes for a minimum 1.5-fold increase compared with the normalized mean across samples.

In this fashion, we identified 151 transcripts specifically over-expressed in sLCs, 118 in sCD14 DCs, 5 in sCD14 ${ }^{+}$DCs, 15 in vLCs, 12 in vCD14 DCs, 6 in vCD14 ${ }^{+}$DCs, 82 in vMøs and 121 in mDCs. In addition, 20 transcripts were specifically over-expressed in sDCs 


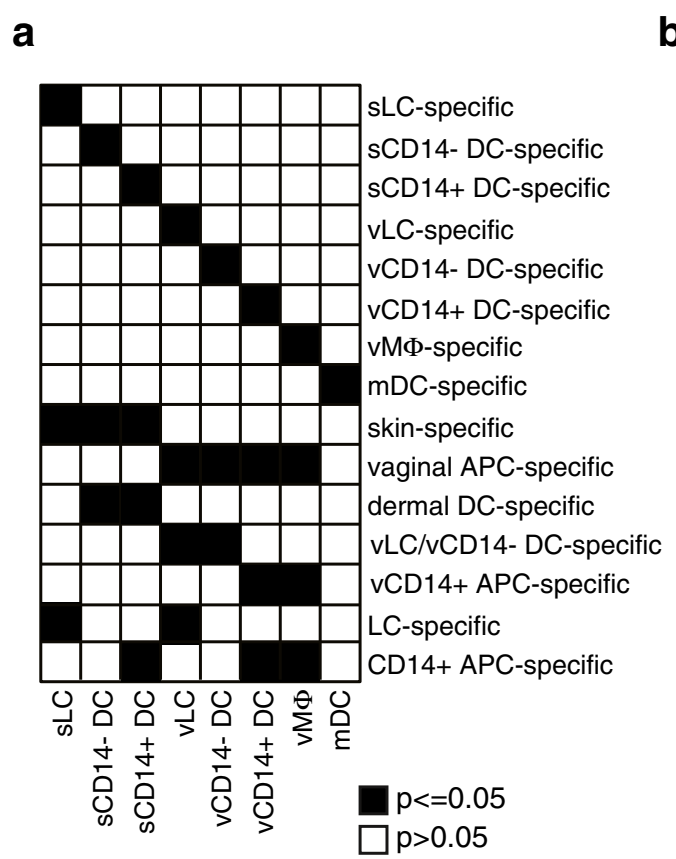

b

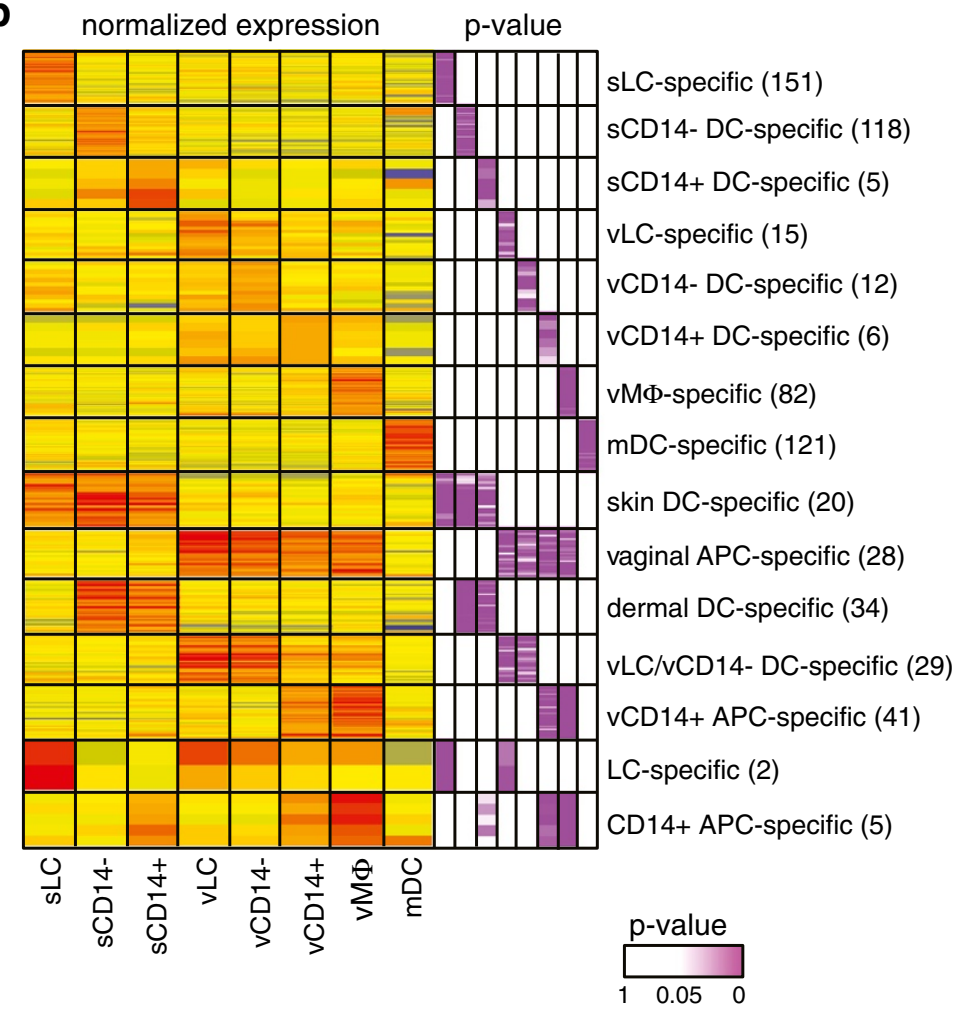

Figure 3 Analysis of variance identifies population-specific transcripts. (a) Heatmap describing the filtering method applied to identify population-specific transcripts. (b) Heatmap representing the normalized expression of transcripts specifically over-expressed in each APC population studied, as well as groups of populations or tissues. $P$-values are represented in purple gradient on the right.

and 28 in vaginal APCs. Population-specific transcripts are represented as knowledge-based Ingenuity Pathway Analysis (IPA) networks in Additional files 4 and 5. These groups of genes can act as potential molecular biomarkers for the APC subsets considered herein.

\section{Two major vaginal APC transcriptional phenotypes}

We then compared the four subsets of vaginal APCs independently of other populations. The 42 samples obtained from vaginal tissue were normalized to the median of all samples. One-way ANOVA identified 1,559 DETs between the four populations. Hierarchical clustering supported the similarity between vLCs and vCD14- DCs as well as between vCD14 $4^{+}$DCs and vMøs (Figure 4a). Tukey's test identified the DETs between vaginal APC populations in a pairwise fashion (Figure 4b). vMøs and vCD14- DCs were most distant, with 653 DETs. Only nine genes separated vLCs and vCD14- DCs. Venn diagram analysis confirmed that the majority of genes (353 out of 370) differentially expressed between vLCs and vMøs were also differentially expressed between vCD14 DCs and vMøs (Figure 4c). Functionally, vLCs and vCD14- DCs displayed similar capacities to induce T-cell responses [5].
The 653 DETs between vCD14- DCs and vMøs are presented as a heatmap (Figure 4d) and further analyzed for regulation and enrichment of biologically relevant pathways by IPA. The predicted regulatory pathways were represented as circular networks with the transcripts on the circumference and the transcription factors and cytokines enriched by in silico IPA network growth in the center. The 157 transcripts over-expressed in vCD14 DCs included transcripts related to antigen processing and presentation (LAMP3, HLA-DOB, HLA-DQB1, CIITA, CD1E), DC maturation (CCR7), interaction with lymphocytes (VCAM1) [24] and Th2 activation molecules (TNFSF4 (OX40L), CCL22) (Figure 4e) [25,26]. These transcripts were connected in silico to molecules involved in the activation of Thelper cells, including CD40LG, IL5, IL13 and IFNG. In addition, vCD14- DCs expressed increased levels of the regulatory $\mathrm{T}$ cell (Treg) inducer IDO1 [27], the antiinflammatory gene $R A M P 1$ [28] and B-cell activation and survival factor TNFSF13B [29]. Conversely, the 496 transcripts over-expressed in vMøs were enriched for Mø markers (CD14, CD163, CD68) and innate proinflammatory molecules (IL8, PLAUR, cathepsins, Fc receptors, CXCLs, complement and bacterial Toll-like receptors (TLRs)). These genes were connected in silico 


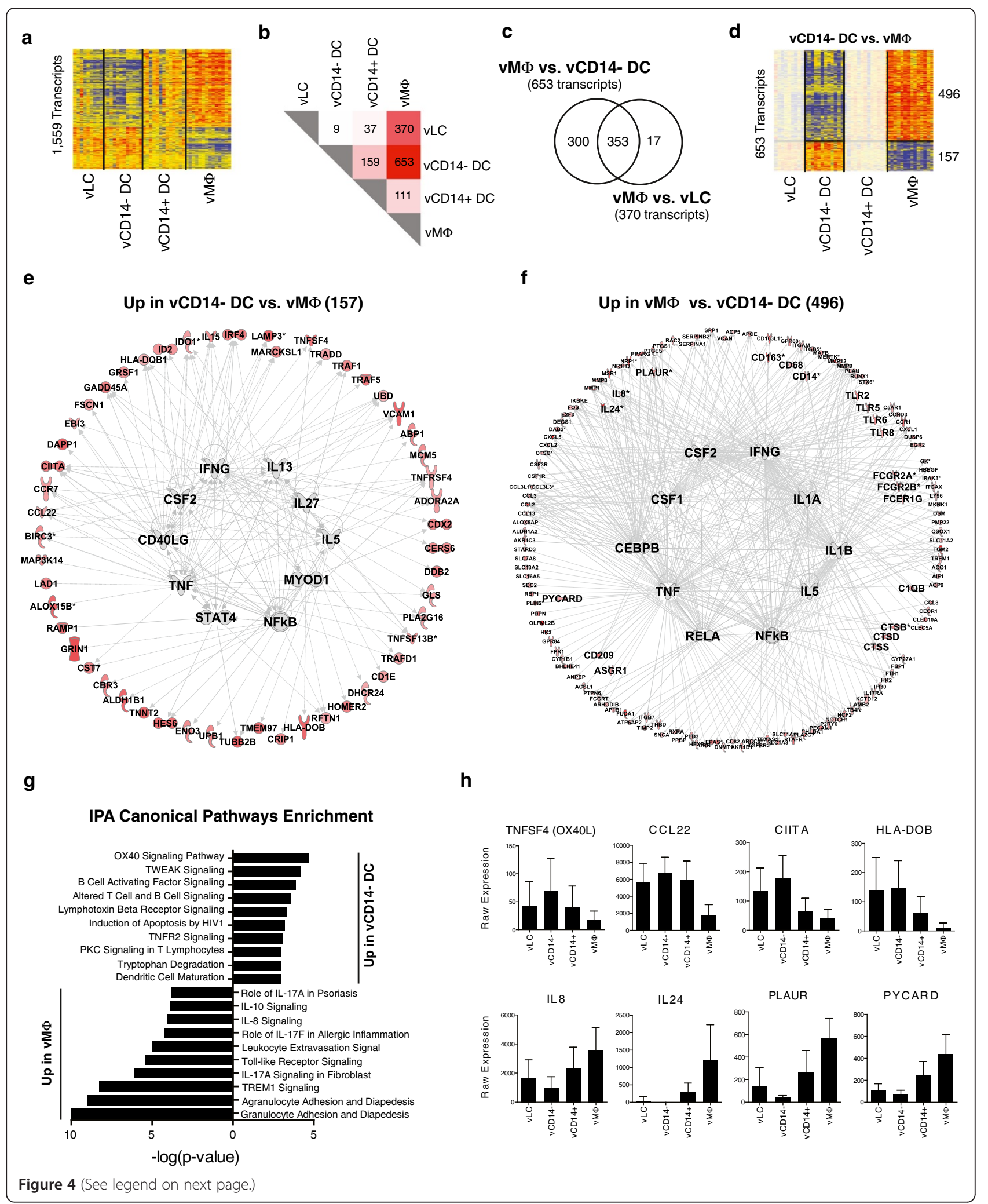


(See figure on previous page.)

Figure 4 Transcriptional analysis of vaginal antigen-presenting cell subsets. (a) Hierarchical clustering (Pearson correlation) of the 1,559 transcripts differentially expressed (one-way ANOVA, $P<0.05$, Benjamini-Hochberg correction) between the four vaginal APC populations. (b) Heatmap representing the results of Tukey's test conducted after ANOVA. (c) Venn diagram of the 653 and 370 transcripts differentially expressed between vCD14 DCs and vMøs and between vLCs and vMøs. (d) Hierarchical clustering (Pearson correlation) of the 653 transcripts differentially expressed between VCD14' DCs and vMøs. (e) IPA network analysis for the 157 transcripts over-expressed in vCD14- DCs compared with vMøs. (f) IPA network analysis for the 496 transcripts over-expressed in vMøs compared with vCD14- DCs. (g) Bar chart representing the IPA canonical pathway enrichment in VCD14- DCs and vMøs. (h) Bar charts representing the batch-corrected expression values for selected transcripts over-expressed in VCD14- DCs (top row) or vMøs (bottom row).

to major inflammatory mediators, including IL1A/B, NFKB/RELA, TNF and colony stimulating factors CSF1 and CSF2 (Figure 4f). In addition, vMøs expressed increased levels of IL24 transcript, which encodes for a cytokine known to promote Th1 polarization [30].

These observations were further supported by IPA canonical pathway and Gene Ontology biological process enrichment analyses, which identified the Th2-inducing OX40 signaling pathway as the most highly enriched pathway in genes over-expressed in vCD14- DCs. Several innate and inflammatory pathways were enriched in vMøs (Figure 4g; Figure S3a in Additional file 6), including signaling of IL8, TLR and the triggering receptor expressed on myeloid cells type-1 (TREM-1), which is an orphan receptor of the immunoglobulin superfamily induced by the DAP12 signaling pathway. TREM-1 activation induces the production of inflammatory cytokines, including IL8, MCP/CCL2 and TNF [31]. The same observations were made when comparing vLCs and vMøs (Figure S3b in Additional file 6; Additional file 7). The batch-corrected expression of transcripts linked to Th2 induction (TNFSF4, CCL22, CIITA, HLADOB) and inflammation (IL8, IL24, PLAUR, PYCARD), which are over-expressed in CD14- vaginal APCs and $\mathrm{CD}_{14}{ }^{+}$vaginal APCs complemented the observations made at the pathway level (Figure 4h).

Altogether, these data suggest that vLCs and vCD14DCs have a transcriptional phenotype oriented towards the activation of Th2 cells. This provides a molecular basis for our previous finding that these populations can differentiate allo-naive $\mathrm{CD}^{+} \mathrm{T}$ cells into $\mathrm{Th} 2$ cells in vitro [5]. In addition, increased expression of IDO and RAMP1 in vCD14- DCs and vLCs suggests that these cells may also have regulatory functions. In contrast, the transcriptionally similar $\mathrm{vCD}^{+} 4^{+} \mathrm{DCs}$ and vMøs are geared for innate immunity, inflammation, pathogenassociated molecular patterns (PAMPs) detection and Th1-type responses.

\section{A regulatory profile in skin epidermal Langerhans cells} We then conducted a similar analysis in skin DC populations, independently of other APC subsets. We identified 3,228 DETs between sLCs, sCD14 $4^{-}$DCs and sCD14 ${ }^{+}$ DCs (Figure 5a). Hierarchical clustering of these transcripts highlighted the transcriptional distance between
sLCs and sCD14 DCs, the latter population being more similar to $\mathrm{sCD}^{+} 4^{+}$DCs. This contrasts with vaginal APCs, where vLCs and vCD14- DCs were almost identical transcriptionally (Figure 4a). Thus, the main separation in the skin results from the epidermal and dermal compartmentalization, which was not the case in the vaginal mucosa (Figure 4). This was further confirmed by Tukey's test, which identified 544 DETs between sLCs and sCD14- DCs and 466 between sLCs and sCD14 ${ }^{+}$ DCs (Figure 5b). Of these, 317 transcripts were shared between the two comparisons as shown by Venn diagram (Figure 5c). Only six transcripts representing four genes were differentially expressed between all three comparisons (CXCL1, CXCL5, IL24 and CTSL1; Additional file 8), which could serve as molecular biomarkers of these three skin DC subsets.

The 544 DETs between sLCs and sCD14- DCs were clustered (Figure 5d) and subjected to IPA network and pathway enrichment analysis. The regulatory network of the 242 transcripts over-expressed in sLCs included sterol regulatory element-binding proteins SREBF1 and SREBF2, two molecules involved in the regulation of lipid and cholesterol biosynthesis [32] (Figure 5e). The transcripts enriched in sLCs were enriched for cholesterol biosynthesis, as well as glutaryl-CoA degradation, tryptophan degradation and the melavonate pathway, three pathways linked to T-cell regulation by DCs and involving the regulatory enzyme indolamine 2,3-dioxygenase (INDO/IDO) [27] (Figure 5g). The transcripts encoding this protein (INDO and IDO1) were overexpressed in sLCs, while both dermal CD14 $4^{-}$and CD14 $4^{+}$ DC subsets were enriched in pro-inflammatory molecules IL1B, TNFA and IL23A (Figure 5h).

Conversely, the 302 transcripts over-expressed in sCD14- DCs (and sCD14 ${ }^{+}$DCs) included innate immunity and pro-inflammatory genes (CCLs, CXCLs, SERPINAs, IL8, IL23A), and were connected to major inflammatory cytokines (IL1, IFNG, TNF) and transcription factors (NFKB, RELA) (Figure 5f). As observed for vCD14 ${ }^{+} \mathrm{DC}$ populations, these transcripts were enriched for pathways linked to innate immunity and inflammation, including TREM1 signaling, Mø function and IL17 signaling (Figure 5g; Additional file 9). This was supported by Gene Ontology enrichment analysis, which identified inflammatory response, defense response and response to stress 


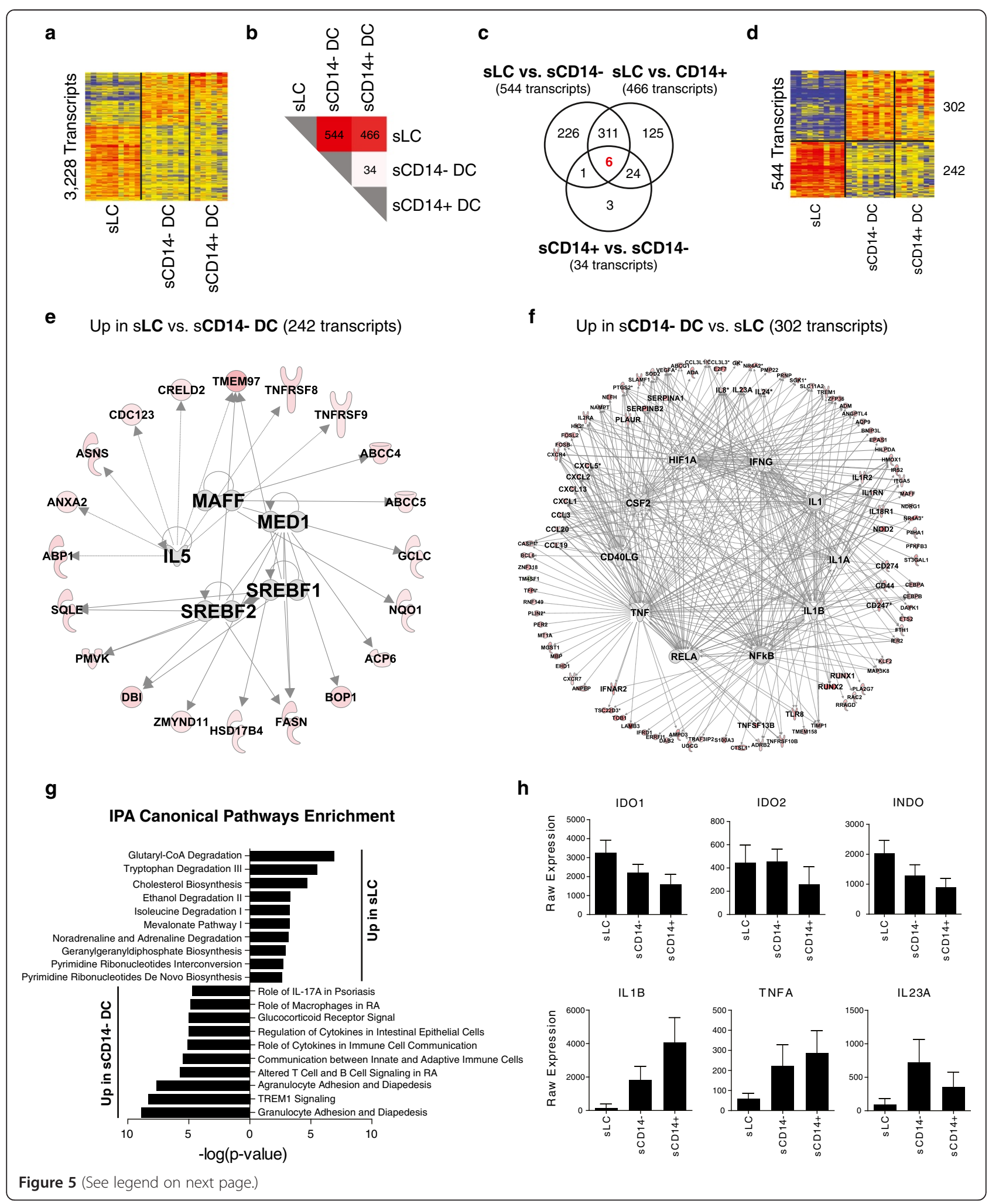


(See figure on previous page.)

Figure 5 Transcriptional analysis of skin dendritic cell subsets. (a) Hierarchical clustering (Pearson correlation) of the 3,228 transcripts differentially expressed (one-way ANOVA, $P<0.05$, Benjamini-Hochberg correction) between the three skin DC populations. (b) Heatmap representing the results of Tukey's test conducted after ANOVA. (c) Venn diagram of the three comparisons conducted during the post hoc test. (d) Hierarchical clustering (Pearson correlation) of the 544 transcripts differentially expressed between sLCs and SCD14 DCs. (e) IPA network analysis for the 242 transcripts over-expressed in sLCs compared with sCD14 DCs. Molecules are colored according to their fold change in the condition represented compared with the median. (f) IPA network analysis for the 302 transcripts over-expressed in SCD14- DCs compared with sLCs. (g) Bar chart representing the IPA canonical pathway enrichment in SLCS and SCD14- DCs. (h) Bar charts representing the batch-corrected expression values for selected transcripts over-expressed in sLCS (top row) or sCD14- DCs (bottom row).

amongst the biological processes enriched in sCD14- DCs (Additional file 10).

Taken together, the three skin DC subsets displayed two major transcriptional phenotypes that were segregated by the tissue compartment in which they localized. Epidermal sLCs displayed a regulatory phenotype, while dermal sCD14 and $\mathrm{sCD}^{-} 4^{+}$DCs displayed an innate immunity and pro-inflammatory phenotype similar to that of vaginal $\mathrm{CD} 14^{+} \mathrm{APCs}$.

\section{Vaginal dendritic cells versus skin dendritic cells}

To further understand the effect of tissue origin on transcriptional phenotype, we compared DC populations from vagina and skin. To this end, $\mathrm{LC}, \mathrm{CD} 14^{-} \mathrm{DC}$ and $\mathrm{CD} 14^{+}$ DC samples from vagina and skin were recombined into a single experiment and analyzed. vMøs were not included as their skin counterparts were not accessible. One-way ANOVA identified 6,599 DETs between the six populations (Figure 6a). Tukey's test identified DETs in a pairwise fashion, with the greatest transcriptional difference between sLCs and vCD14 ${ }^{+}$DCs (Figure $6 \mathrm{~b}$ ). The DETs between sLCs and vLCs, sCD14 and vCD14 DCs and $\mathrm{sCD}_{1}{ }^{+}$and $\mathrm{vCD}^{+} 4^{+} \mathrm{DCs}$ are clustered and represented as heatmaps in Figure 6c.

Venn diagrams identified overlap between the three comparisons, as well as DETs in each comparison (Figure 6d). These included 12 transcripts over-expressed in vagina and 8 transcripts over-expressed in skin in all three comparisons (Figure 6e), providing a 20 transcript tissue-specific signature. As a non-APC tissue control, transcriptional profiles for vaginal HLA-DR ${ }^{-}$cells were also obtained (seven biological replicates). Among the genes enriched in vaginal tissue, LTB (lymphotoxin- $\beta$ ) and CD24 were also overexpressed in HLA-DR ${ }^{-}$vaginal cells. Lymphotoxin signaling is important for the development and maintenance of lymphoid tissue structure, including Peyer's patches in the mucosa [33], and is also associated with the ability of mucosal DCs to regulate IgA production by plasma cells [34]. CD24 expressed on DCs acts as a co-stimulatory molecule for Th17 cells [35] and cytotoxic T lymphocyte responses [36]. In addition, AXL, a member of the TAM receptor tyrosine kinase family involved in regulation of TLR and interferon-induced inflammatory cascades, was selectively over-expressed in $\mathrm{HLA}-\mathrm{DR}^{+}$vaginal populations. sDCs were enriched for CYTIP, a molecule involved in DC motility [37] that also displays regulatory functions in mouse DCs [38]. The DETs in each of the three comparisons are hierarchically clustered and displayed in Additional files 11, 12 and 13.

We also compared vDCs and sDCs by $t$-test, grouping cells by tissue, and including HLA-DR vaginal cells as control. We found 1,007 DETs between sDCs and vDCs, with 640 over-expressed in sDCs and 367 overexpressed in vDCs (Figure S11a in Additional file 14). The transcripts over-expressed in sDCs were enriched for metabolic processes, including metabolism of nitrogen compounds and RNA, while the transcripts overexpressed in vDCs were enriched for immune-related biological processes, including defense response, phagocytosis and cell adhesion (Figure S11b in Additional file 14). We further compared the 367 transcripts overexpressed in vDCs (compared with sDCs) to HLA-DR ${ }^{-}$ vaginal cells. Of these, 88 were similarly expressed, 123 were under-expressed and 156 were over-expressed in HLA-DR ${ }^{-}$vaginal cells. The genes over-expressed in vaginal HLA-DR ${ }^{-}$cells compared with vaginal HLA-DR ${ }^{+}$ cells included many transcripts expressed in epithelial cells, such as keratins, defensins and serpinases, with network connections to inflammatory cytokines such as IL17, IL1 and type I and II interferon (Figure S11c in Additional file 14). The 123 transcripts over-expressed in HLA-DR ${ }^{+}$DCs were enriched for CLEC10A, OLR1, cathepsins, CD1s and Mø markers such as CD163.

Globally, tissue comparisons yielded more transcripts linked to immune response and inflammation in vDCs, while sDCs exhibited a metabolism fingerprint.

\section{Pattern-recognition receptor expression in vaginal APC subsets}

To further understand the phenotype of vaginal APC subsets and the immunology of the human vagina, we analyzed the expression levels of pattern-recognition receptors, including C-type lectin-like receptors (LLRs) and TLRs, and compared them with those of skin and blood DC subsets. Vaginal APC populations expressed increased levels of CLEC5A, CLEC4F, CLEC4A, CLEC2B, CLEC16A, OLR1, CLEC10A, and CD209 compared with other LLRs (Additional file 15). Consistent with the transcriptional 


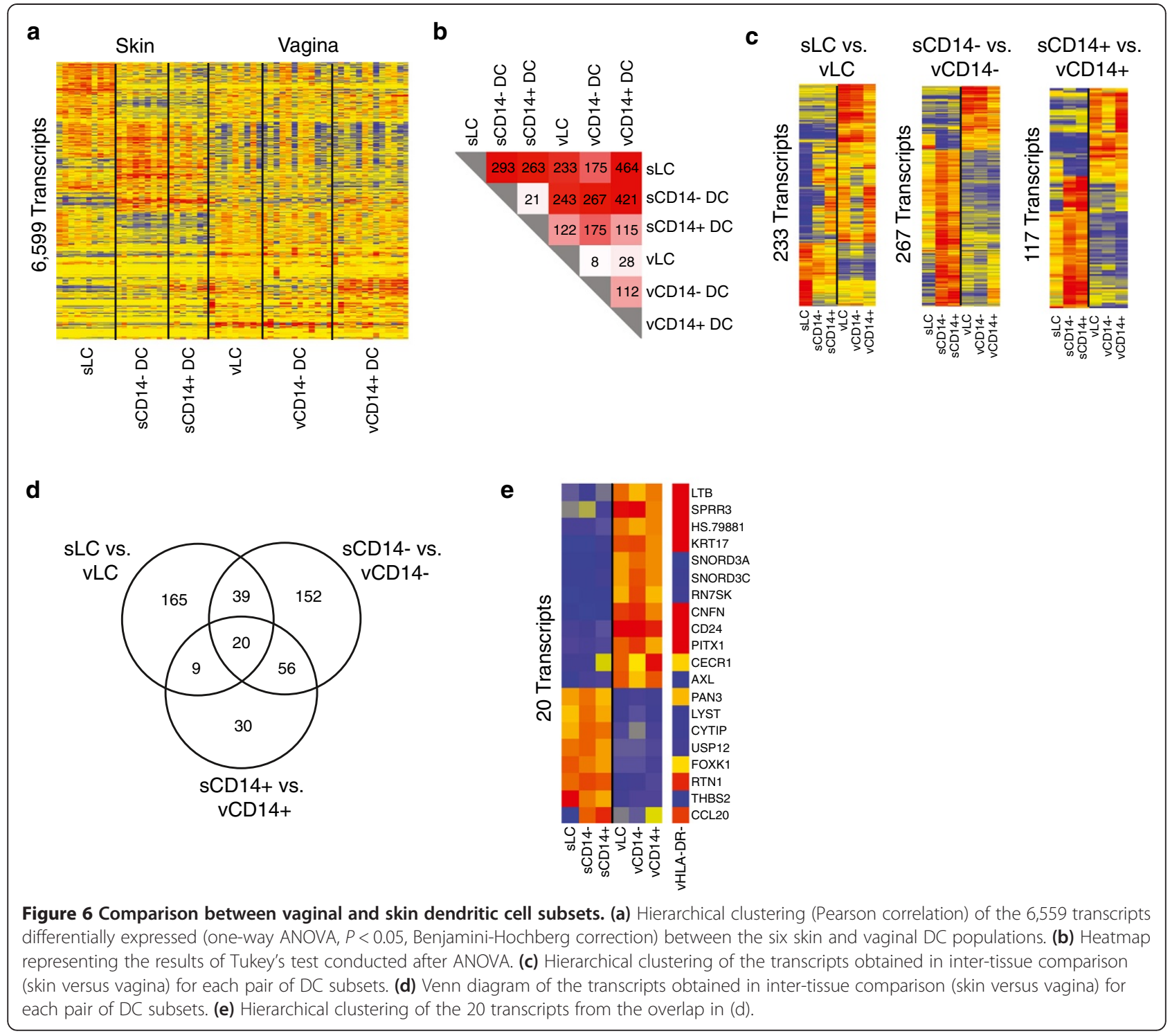

phenotypes of vaginal APCs (Figure 4), vLCs were close to vCD14- DCs while vCD14 ${ }^{+}$DCs were close to vMøs in terms of expression levels of the eight LLRs. Both CD209 and OLR1 were highly expressed especially in vCD14 ${ }^{+}$ DCs and vMøs. vCD14 ${ }^{+}$DCs and vMøs expressed higher levels of CD209 and OLR1 than sCD14. DCs. In addition, CLEC5A was expressed in vLCs but not sLCs. Similarly, vCD14- DCs expressed CLEC4F, while sCD14 DCs showed no or minimal expression of CLEC4F. Compared with vaginal APCs and sDCs, blood $\mathrm{mDCs}$ expressed increased levels of CLEC2B, CLEC10A, and CLEC12A, but decreased levels of CD209, CLEC5A, and CLEC4F. Furthermore, batch-corrected expression analysis identified three additional lectins that were over-expressed in vCD14 ${ }^{+}$DCs and vMøs: CLEC2B, CLEC5A and LGALS8 (Additional files 15 and 16). Conversely, only CLEC16A was over-expressed in vLCs and vCD14- DCs.
In contrast to LLRs, vDC subsets and their skin counterparts expressed similar levels of TLRs, MDA5, and RIG-I (Additional file 17). However, $\mathrm{CD} 14^{+}$populations, particularly vMøs, expressed increased levels of bacterialsensing TLRs (TLR2, TLR5, TLR6, TLR8).

We next assessed the expression levels of LLRs and other receptors that have been previously tested for vaccines targeting in vivo DCs [21,39-43]. CD209 and CLEC10A, OLR1, and CLEC4A, but not CLEC13B, were detected in all four vaginal APC subsets with increased expression of CD209, CLEC10A, and CLEC4A in vMøs (Figure 7a; Additional file 18). The expression of DC-SIGN, DCASGPR, and LOX-1 was confirmed on $\mathrm{vCD}^{+} 4^{+}$DCs and vMøs at the protein level by flow cytometry (Figure 7b) and in situ by immunofluorescence (Figure 7c). LOX-1 was also expressed at low levels on vCD14 DCs (Figure 7b,c). Transcriptional levels of CD207 were low in all vaginal APC 
a

$\square$ vLC $\square$ vCD14- $\square$ vCD14+ $\square$ vM $\Phi$

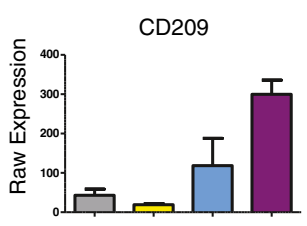

OLR1

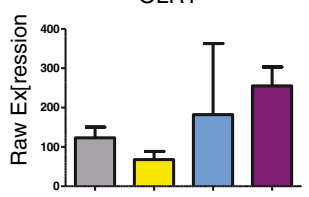

CLEC10A

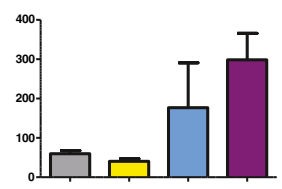

CLEC4A

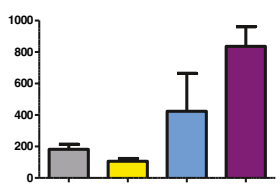

C

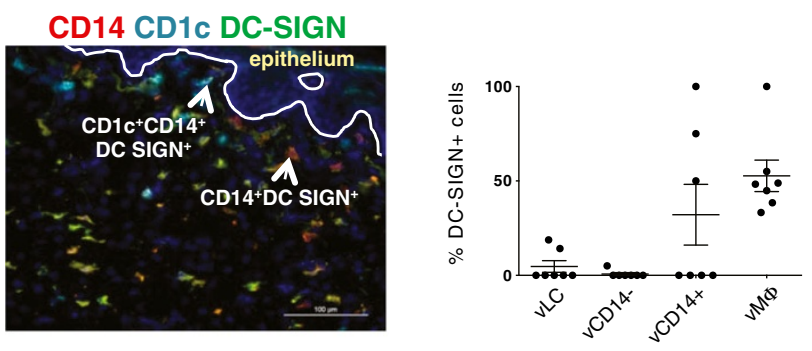

CD14 CD1C DC-ASGPR
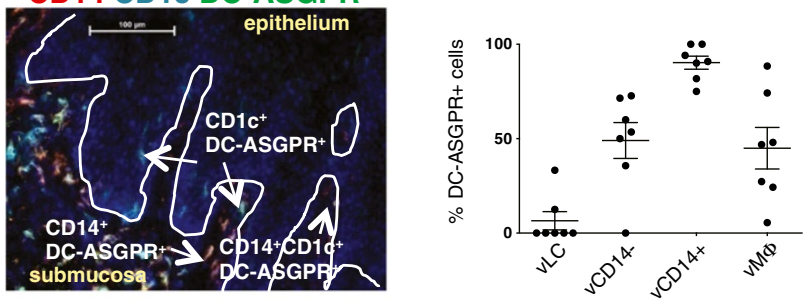

b

\section{CD14 CD1c LOX-1}
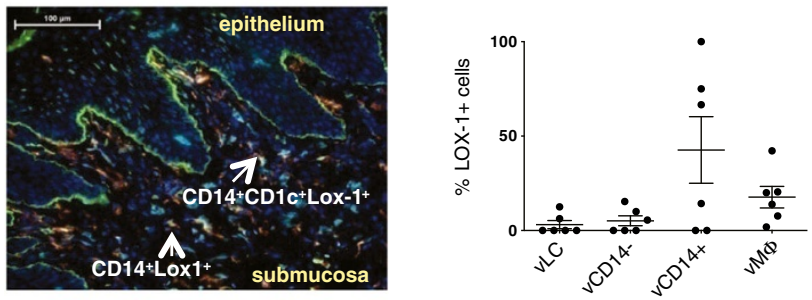

(CLEC10A)
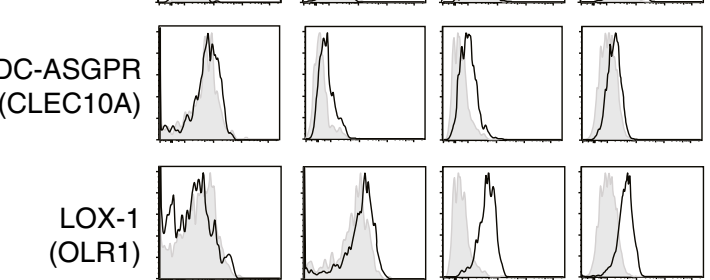

(CLEC4A)
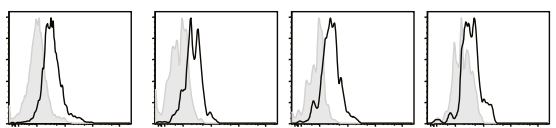

DEC-205 (CLEC13B)
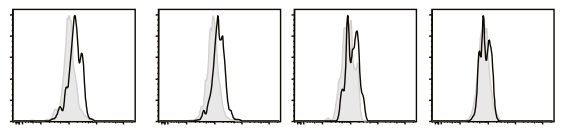

CD40
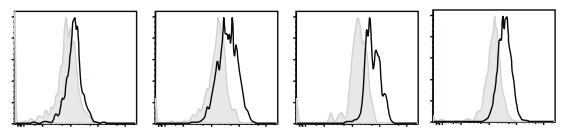

isotype
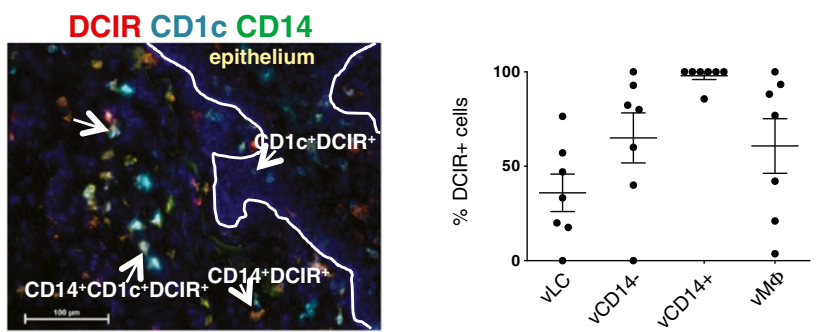

CD207 CD1c DEC-205
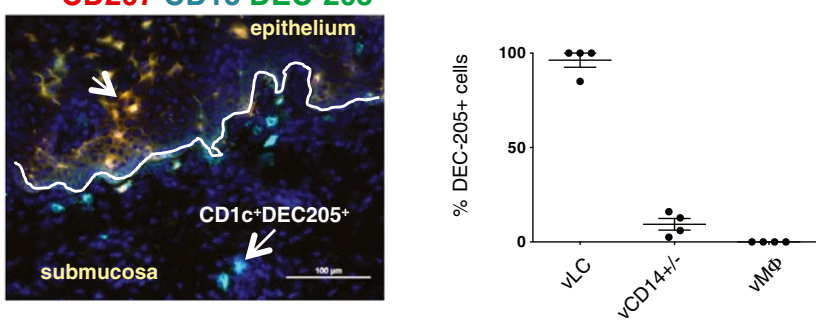

Figure 7 Surface receptor expression on vaginal antigen-presenting cells. (a) Transcriptional levels of CD209 (DC-SIGN), OLR1 (LOX-1), CLEC10A (DC-ASGPR) and CLEC4A (DCIR) in the four vaginal APC populations. Bar charts represent the mean \pm standard deviation of batch-corrected expression data. (b) Fluorescence-activated cell sorting (FACS) analysis of CD209, DC-ASGPR, LOX-1, DCIR, DEC-205 and CD40 expression on the surface of vaginal APC subsets. Vaginal cell suspensions were stained with the indicated antibodies and gated as described in Figure 1b. Gray histograms represent isotype controls. Data are representative of 10 donors. (c) Frozen tissue sections were stained for (i) DC-SIGN, DC-ASGPR or LOX-1 (green), CD1C (light blue); and CD14 (red); or (ii) DCIR (red), CD1c (light blue) and CD14 (green); or (iii) DEC-205 (green), CD1c (light blue) and CD207 (red) (DAPI (dark blue), ×20, the horizontal bar represents $100 \mu$ m). Data are representative of four to eight independent experiments (left panel) or combined (right panel). Isotype controls are presented in Additional file 18. 
subsets, despite detectable protein levels on the surface of vLCs (Figure 1b), as previously reported [5,6]. DC-ASGPR was detected in a few vLCs and approximately 50\% (49.06 \pm 25) of vCD14 DCs (Figure 7b, c). DCIR was detected on all APC subsets by immunofluorescence and flow cytometry (Figure 7c). DEC-205 was detected on LCs and some submucosal DCs by immunofluorescence and flow cytometry, despite undetectable transcriptional expression. CD40 transcriptional levels were low in all vaginal APC subsets (not shown), although CD40 was detected on all migrated vaginal APC subsets by flow cytometry (Figure 7b).

\section{Expression of inflammatory mediators in vaginal APC subsets}

Finally, we analyzed the expression of cytokines, chemokines and their receptors. The results are summarized in Additional file 19. Dermal DCs and vaginal $\mathrm{CD} 14^{+}$populations displayed increased levels of neutrophil chemoattractants CXCL1, CXCL2 and CXCL5 (Additional file 20), as well as pro-inflammatory cytokines IL1A, IL1B, IL24, TNF and IL8 (Additional file 21). All sDC populations expressed higher levels of IL23A, while vaginal populations displayed increased IL32, and $\mathrm{mDCs}$ displayed increased IL12A and CXCL10, suggesting tissue-specific capacities to polarize T-helper responses. Dermal DCs expressed higher levels of IL1R1, IL1R2 and IL18R, which may polarize them to respond to products of the inflammasome, while vMøs were enriched in IL10RB and IL17RA, two receptors involved in defense against extracellular pathogens. Interestingly, sLCs expressed increased levels of several CKLF-like MARVEL transmembrane domaincontaining proteins (CMTM4, CMTM6, CMTM8), which constitute a novel family of chemokine receptors about which little is known.

\section{Discussion}

We report the first transcriptional characterization of four human vaginal APC subsets by microarray. We compared these transcriptional profiles with those of three skin DC populations and blood mDCs. Combining unsupervised, pathway-level and transcript-level analyses, we identified tissue- and population-specific transcripts, as well as whole molecular pathways that potentially control the biological functions of APCs in the human vaginal mucosa and the skin $[3,5,44]$. This database of 87 microarray samples obtained from human vagina, skin and blood provides an important resource to understand tissue-specific immunity and guide the rational development of microbial vaccines.

Unsupervised analysis enabled the measurement of the transcriptional separation between APC populations in an unbiased fashion. vLCs were similar to vCD14- DCs, while vCD $14^{+}$DCs were similar to vMøs, suggesting that CD14 expression can be used to differentiate between two main APC groups with distinct fingerprints. The global transcriptional distance between populations is probably best expressed by PCA (Figure 2d), and can be confirmed by Tukey's post hoc test (Figure 2e). Tukey's post hoc test is a stringent test identifying pairwise differences within the populations considered for ANOVA. Because of the stringency of the test, the number of genes differentially expressed between two populations is affected by the number of samples and variability within groups. vCD14 ${ }^{+}$DCs and vLCs displayed a higher degree of variability across donors than vMøs, thereby explaining lower numbers of genes detected, despite more similar profiles between vCD14. $\mathrm{DCs}$ and Møs. In fact, both $\mathrm{CD}_{1} 4^{+}$populations presented an innate inflammatory profile with increased expression of pattern-recognition receptors, consistent with less mature populations. The CD14 populations were enriched for T-cell co-stimulation and antigen-presentation transcripts, consistent with a more mature profile. The transcriptional proximities between CD14- APCs and CD14 ${ }^{+}$ APCs are concordant with the functional capacities we previously described, where vLCs and vCD14 DCs polarize $\mathrm{CD} 4^{+} \mathrm{T}$ cells towards the Th2 phenotype [5]. In this context, the increase in OX40L (TNFSF4), CCL22 and MHC class II-related transcripts (CIITA and HLADRs) in CD14 APC populations suggests an OX40Ldependent mechanism of Th2 induction. Interestingly, sDCs did not show the same transcriptional separation based on expression of CD14. Their transcriptional phenotype separated mainly based on physical location, where the two dermal populations displayed a profile distinct from that of sLCs. Finally, it is important to note that the individual APC populations we describe in this manuscript may contain additional levels of heterogeneity. For example, we have previously shown that both CD14 and CD14 ${ }^{+}$LP-DCs could be further separated into two subpopulations based on CD1a expression $[5,6]$. Further studies, such as single-cell RNA sequencing of populations of interest, are warranted to further characterize the heterogeneity of these populations.

When comparing transcriptional fingerprints between tissues, we observed that sDCs were enriched for metabolic pathways, while vDCs were enriched for immunerelated networks. Although the female genital tract is considered an immune-privileged site [10-12], this observation, along with the data from our previous study, [5] supports that DCs in the human vaginal mucosa can elicit immune responses, as previously observed in mice [45]. These findings have important implications for the rational design of mucosal vaccines against sexually transmitted pathogens.

In addition, the transcriptional profiling of skin and vaginal APCs enabled us to formulate new hypotheses on the mechanisms controlling some of the known functions 
of these subsets. For instance, transcripts encoding IDO1/ INDO, a molecule involved in DC-dependent induction of Tregs, were significantly overexpressed in sLCs compared with other sDCs (Figure 5h). These data support recent studies showing the induction of Tregs by sLCs in the absence of foreign antigen stimulation $[44,46]$. Therefore, these factors should be considered in the development of vaccines that elicit potent immunity in the vaginal mucosa.

The differences observed between tissues may also be influenced by the composition of the microbiome from each tissue. The vaginal microbiome is highly enriched in lactobacilli [47], while the skin microbiome is enriched for staphylococci and actinobacteria [15,48]. In fact, it was shown that microbiota can influence immunity by triggering expression of C-type lectins [49] or controlling the Th1/Th2 balance [50]. The interactions between the microbiome and DC functions in different tissues and the outcomes of immune responses at steady state and during infections need to be further studied.

Finally, we used these data to formulate testable hypotheses regarding the expression of various receptors on the surface of each vaginal APC population. As vaginal $\mathrm{CD} 14^{+}$cells displayed a more immature and innate phenotype, it was logical to find that transcripts for receptors that could be used as targeting molecules for antibody-conjugated vaccines (such as C-type lectins) were globally enriched in these populations, particularly in vMøs. Of the known lectins, only CLEC16A was transcriptionally enriched in vLCs and vCD14- DCs. Interestingly, this putative immuno-receptor, which belongs to a gene complex involving CIITA and SOCS1, is linked to autoimmune disorders such as multiple sclerosis and rheumatoid arthritis [51,52].

Our study has several limitations. First, the majority of skin samples were obtained from individuals with high body mass index undergoing cosmetic surgeries. Therefore, we cannot exclude that the lipid-rich environment of the skin affects the transcriptional profiles observed. Although inflamed vaginal tissues were excluded in this study, a large fraction of samples were from patients who underwent pelvic surgeries. These challenges are common in the study of human immunology using surgical tissue samples. Another major limitation is the in vivo relevance of the data generated in vitro. The data generated with human tissue samples still needs to be validated in vivo. In this respect, the surface biomarkers of the vaginal APC subsets characterized in this study are important findings and clinically relevant because we can now design mucosal vaccines that can target specific receptors that are expressed on specific subsets of the vaginal APCs. Vaccines targeting the proper subsets of DCs in conjunction with appropriate adjuvants have proven an efficient strategy to elicit potent immunity [21,40-43].
To conclude, this study provides new insights on the molecular mechanisms that regulate the functions of vaginal APC subsets. The identification of population-specific biomarkers combined with an understanding of major functional characteristics of each APC population in the vaginal mucosa will be important for the development of targeted vaccines against sexually transmitted pathogens, as well as cancers, in the female genital tract.

\section{Conclusions}

We generated a transcriptional dataset of 87 microarray samples spanning eight APC populations across human vagina, skin and blood. Complementary transcript and network-level analyses of these data, combined with in situ immunohistochemistry of major pattern recognition receptors in the vaginal mucosa, permitted the phenotypic and functional characterization of these populations. By comparing vagina, skin and blood, we found that APC genomic fingerprints are significantly influenced by the tissue of origin, revealing tissue-specific microenvironments. Nonetheless, CD14 ${ }^{+}$APCs from both vagina and skin are geared towards innate immunity and pro-inflammatory responses, whereas CD14- DCs, particularly sLCs, vLCs, and vCD14DCs, display both Th2-inducing and regulatory phenotypes. These data will help the further characterization of human tissue APC lineages and will guide the design of mucosal vaccines against sexually transmitted pathogens.

\section{Data access}

The dataset described in this manuscript is deposited in the NCBI Gene Expression Omnibus [53] (GEO series accession number GSE54480). Both background-subtracted and batch-corrected expression datasets are presented.

\section{Additional files}

Additional file 1: Figure S16. The expression of CD1a on the skin DCs. Additional file 2: Figure S17. Results of batch correction.

Additional file 3: Figure S1. Heatmap showing DETs between the eight populations.

Additional file 4: Figure S2. Population-specific transcript networks. Additional file 5: Table S1. Lists the expression of population-specific transcripts.

Additional file 6: Figure S3. Pathway enrichment scores in vaginal DC subsets.

Additional file 7: Figure S4. Results of the comparisons between vaginal LC and macrophages.

Additional file 8: Figure S5. Transcripts specific for skin APC populations.

Additional file 9: Figure S6. Results of comparisons between $S L C s$ and SCD $14^{+}$DCs.

Additional file 10: Figure S7. Pathways enrichment scores in skin LCs and skin CD14- DCs.

Additional file 11: Figure S8. DETs between skin and vaginal APC subsets. 
Additional file 12: Figure S9. DETs between skin and vaginal APC subsets.

Additional file 13: Figure S10. DETs between skin and vaginal APC subsets.

Additional file 14: Figure S11. IPA analysis of DETs in skin and vagina. Additional file 15: Figure S12. Raw transcriptional expression of C-type lectins, TLRs, chemokines, cytokines and their receptors in all APC subsets.

Additional file 16: Table S2. Expression of surface molecules, cytokines and chemokines on all APC subsets.

Additional file 17: Figure S13. Raw transcriptional expression of C-type lectins, TLRs, chemokines, cytokines and their receptors in all APC subsets.

Additional file 18: Figure S18. Immunofluorescence staining of lectin receptors on human vagina.

Additional file 19: Table S3. Expression of surface molecules, cytokines and chemokines on all APC subsets.

Additional file 20: Figure S14. Raw transcriptional expression of C-type lectins, TLRs, chemokines, cytokines and their receptors in all APC subsets.

Additional file 21: Figure S15. Raw transcriptional expression of C-type lectins, TLRs, chemokines, cytokines and their receptors in all APC subsets.

\section{Abbreviations}

ANOVA: analysis of variance; APC: antigen-presenting cell; DC: dendritic cell; DET: differentially expressed transcript; IL: interleukin; IPA: Ingenuity Pathway Analysis; IRB: institutional review board; LC: Langerhans cell; LLR: lectin-like receptor; LP: lamina propria; mDC: myeloid dendritic cell; Mø: macrophage; PCA: principal component analysis; PVCA: principal variance component analysis; sLC: skin Langerhans cell; TLR: Toll-like receptor; TNF: tumor necrosis factor; Treg: regulatory T cell.

\section{Competing interests}

The authors declare that they have no competing interests.

\section{Authors' contributions}

DD designed the study, collected and processed samples, interpreted data and wrote the manuscript. RB performed all analysis, interpreted data and wrote the manuscript. JG performed immunofluorescence staining and helped with vagina tissue processing. LTS and JPB helped with skin sample processing. SZ and GZ provided reagents. SH and NB helped with the analysis. JS, MC and MB provided clinical samples. GZ, LTS, JRU, VP, NB, MC and $M B$ critically reviewed the manuscript. SO designed the study, interpreted data, supervised the study and wrote the manuscript. All authors read and approved the final manuscript.

\section{Acknowledgments}

We thank the Sample, Genomics, FACS, Cell Processing, and Imaging Cores at BIIR. We thank Dr Carson Harrod and Mr Jerome Ellis (BIIR) for proofreading this manuscript. We also thank Dr Michael Ramsay for supporting our program. This study was funded by 1RC1Al087379-01, U19 Al057234 (NIH) and the Baylor Health Care System Foundation.

\section{Author details}

'Baylor Institute for Immunology Research, 3434 Live Oak St, Dallas, TX 75204, USA. ${ }^{2}$ Department of Obstetrics and Gynecology, Baylor University Medical Center, 3600 Gaston Ave, Dallas, TX 75246, USA.

Received: 11 June 2014 Accepted: 28 October 2014

Published online: 25 November 2014

\section{References}

1. Steinman RM, Hawiger D, Nussenzweig MC: Tolerogenic dendritic cells. Annu Rev Immunol 2003, 21:685-711.

2. Fujita H, Nograles KE, Kikuchi T, Gonzalez J, Carucci JA, Krueger JG: Human Langerhans cells induce distinct IL-22-producing CD4+ T cells lacking IL-17 production. Proc Natl Acad Sci U S A 2009, 106:21795-21800.

3. Klechevsky E, Morita R, Liu M, Cao Y, Coquery S, Thompson-Snipes L, Briere F, Chaussabel D, Zurawski G, Palucka AK, Reiter Y, Banchereau J, Ueno H:
Functional specializations of human epidermal Langerhans cells and CD14+ dermal dendritic cells. Immunity 2008, 29:497-510.

4. Mathers AR, Janelsins BM, Rubin JP, Tkacheva OA, Shufesky WJ, Watkins SC, Morelli AE, Larregina AT: Differential capability of human cutaneous dendritic cell subsets to initiate Th17 responses. J Immunol 2009, 182:921-933.

5. Duluc D, Gannevat J, Anguiano E, Zurawski S, Carley M, Boreham M, Stecher J, Dullaers M, Banchereau J, Oh S: Functional diversity of human vaginal APC subsets in directing T-cell responses. Mucosal Immunol 2013, 6:626-638.

6. Duluc D, Gannevat J, Joo H, Ni L, Upchurch K, Boreham M, Carley M, Stecher J, Zurawski G, Oh S: Dendritic cells and vaccine design for sexually-transmitted diseases. Microb Pathog 2013, 58:35-44.

7. Iwasaki A: Mucosal dendritic cells. Annu Rev Immunol 2007, 25:381-418.

8. Iwasaki A: Antiviral immune responses in the genital tract: clues for vaccines. Nat Rev Immunol 2010, 10:699-711.

9. Starnbach MN, Roan NR: Conquering sexually transmitted diseases. Nat Rev Immunol 2008, 8:313-317.

10. Mestecky J, Moldoveanu Z, Russell MW: Immunologic uniqueness of the genital tract: challenge for vaccine development. Am J Reprod Immunol 2005, 53:208-214.

11. Russell MW, Mestecky J: Humoral immune responses to microbial infections in the genital tract. Microbes Infect 2002, 4:667-677.

12. Russell MW, Mestecky J: Tolerance and protection against infection in the genital tract. Immunol Invest 2010, 39:500-525

13. Geijtenbeek TB, Gringhuis SI: Signalling through C-type lectin receptors: shaping immune responses. Nat Rev Immunol 2009, 9:465-479.

14. Robinson MJ, Sancho D, Slack EC, LeibundGut-Landmann S, Reis e Sousa C: Myeloid C-type lectins in innate immunity. Nat Immunol 2006, 7:1258-1265.

15. Grice EA, Segre JA: The skin microbiome. Nat Rev Microbiol 2011, 9:244-253.

16. Kaushic $\mathrm{C}$ : The role of the local microenvironment in regulating susceptibility and immune responses to sexually transmitted viruses in the female genital tract. J Reprod Immunol 2009, 83:168-172.

17. Woodrow KA, Bennett KM, Lo DD: Mucosal vaccine design and delivery. Annu Rev Biomed Eng 2012, 14:17-46.

18. Heng TS, Painter MW, Immunological Genome Project C: The Immunological Genome Project: networks of gene expression in immune cells. Nat Immunol 2008, 9:1091-1094.

19. Miller JC, Brown BD, Shay T, Gautier EL, Jojic V, Cohain A, Pandey G, Leboeuf M, Elpek KG, Helft J, Hashimoto D, Chow A, Price J, Greter M, Bogunovic M, Bellemare-Pelletier A, Frenette PS, Randolph GJ, Turley SJ, Merad M, Immunological Genome Consortium: Deciphering the transcriptional network of the dendritic cell lineage. Nat Immunol 2012, 13:888-899.

20. Watchmaker PB, Lahl K, Lee M, Baumjohann D, Morton J, Kim SJ, Zeng R, Dent A, Ansel KM, Diamond B, Hadeiba H, Butcher EC: Comparative transcriptional and functional profiling defines conserved programs of intestinal DC differentiation in humans and mice. Nat Immunol 2014, 15:98-108.

21. Li D, Romain G, Flamar AL, Duluc D, Dullaers M, Li XH, Zurawski S, Bosquet N, Palucka AK, Le Grand R, O'Garra A, Zurawski G, Banchereau J, Oh S: Targeting self- and foreign antigens to dendritic cells via DC-ASGPR generates IL-10-producing suppressive CD4+ T cells. J Exp Med 2012, 209:109-121.

22. sva R/Bioconductor package [http://www.bioconductor.org/packages/ release/bioc/html/sva.html]

23. pvca R/Bioconductor package [http://www.bioconductor.org/packages/ release/bioc/html/pvca.html]

24. Elices MJ, Osborn L, Takada Y, Crouse C, Luhowskyj S, Hemler ME, Lobb RR: VCAM-1 on activated endothelium interacts with the leukocyte integrin VLA-4 at a site distinct from the VLA-4/fibronectin binding site. Cell 1990, 60:577-584.

25. Andrew DP, Chang MS, McNinch J, Wathen ST, Rihanek M, Tseng J, Spellberg JP, Elias CG 3rd: STCP-1 (MDC) CC chemokine acts specifically on chronically activated Th2 lymphocytes and is produced by monocytes on stimulation with Th2 cytokines IL-4 and IL-13. J Immunol 1998, 161:5027-5038.

26. Ito T, Wang YH, Duramad O, Hori T, Delespesse GJ, Watanabe N, Qin FX, Yao Z, Cao W, Liu YJ: TSLP-activated dendritic cells induce an inflammatory T helper type 2 cell response through OX40 ligand. J Exp Med 2005, 202:1213-1223. 
27. Munn DH, Zhou M, Attwood JT, Bondarev I, Conway SJ, Marshall B, Brown C, Mellor AL: Prevention of allogeneic fetal rejection by tryptophan catabolism. Science 1998, 281:1191-1193.

28. Tsujikawa K, Yayama K, Hayashi T, Matsushita H, Yamaguchi T, Shigeno T, Ogitani Y, Hirayama M, Kato T, Fukada S, Takatori S, Kawasaki H, Okamoto H, Ikawa M, Okabe M, Yamamoto H: Hypertension and dysregulated proinflammatory cytokine production in receptor activity-modifying protein 1-deficient mice. Proc Natl Acad Sci U S A 2007, 104:16702-16707.

29. Mackay F, Schneider P: Cracking the BAFF code. Nat Rev Immunol 2009, 9:491-502.

30. Caudell EG, Mumm JB, Poindexter N, Ekmekcioglu S, Mhashilkar AM, Yang $\mathrm{XH}$, Retter MW, Hill P, Chada S, Grimm EA: The protein product of the tumor suppressor gene, melanoma differentiation-associated gene 7, exhibits immunostimulatory activity and is designated IL-24. J Immunol 2002, 168:6041-6046.

31. Colonna M: TREMs in the immune system and beyond. Nat Rev Immunol 2003, 3:445-453.

32. Horton JD, Goldstein JL, Brown MS: SREBPs: activators of the complete program of cholesterol and fatty acid synthesis in the liver. J Clin Invest 2002, 109:1125-1131.

33. Gommerman JL, Browning JL: Lymphotoxin/light, lymphoid microenvironments and autoimmune disease. Nat Rev Immunol 2003, 3:642-655.

34. Kang HS, Chin RK, Wang Y, Yu P, Wang J, Newell KA, Fu YX: Signaling via LTbetaR on the lamina propria stromal cells of the gut is required for IgA production. Nat Immunol 2002, 3:576-582.

35. Schlitzer A, McGovern N, Teo P, Zelante T, Atarashi K, Low D, Ho AW, See P, Shin A, Wasan PS, Hoeffel G, Malleret B, Heiseke A, Chew S, Jardine L, Purvis HA, Hilkens CM, Tam J, Poidinger M, Stanley ER, Krug AB, Renia L, Sivasankar B, Ng LG, Collin M, Ricciardi-Castagnoli P, Honda K, Haniffa M, Ginhoux F: IRF4 transcription factor-dependent CD11b + dendritic cells in human and mouse control mucosal IL-17 cytokine responses. Immunity 2013, 38:970-983

36. Kim TS, Gorski SA, Hahn S, Murphy KM, Braciale TJ: Distinct dendritic cell subsets dictate the fate decision between effector and memory CD8(+) T cell differentiation by a CD24-dependent mechanism. Immunity 2014, 40:400-413.

37. Theodoridis AA, Eich C, Figdor CG, Steinkasserer A: Infection of dendritic cells with herpes simplex virus type 1 induces rapid degradation of CYTIP, thereby modulating adhesion and migration. Blood 2011, 118:107-115.

38. Heib V, Sparber F, Tripp CH, Ortner D, Stoitzner P, Heufler C: Cytip regulates dendritic-cell function in contact hypersensitivity. Eur J Immunol 2012, 42:589-597.

39. Bonifaz LC, Bonnyay DP, Charalambous A, Darguste DI, Fujii S, Soares H, Brimnes MK, Moltedo B, Moran TM, Steinman RM: In vivo targeting of antigens to maturing dendritic cells via the DEC-205 receptor improves T cell vaccination. J Exp Med 2004, 199:815-824

40. Cohn L, Chatterjee B, Esselborn F, Smed-Sorensen A, Nakamura N, Chalouni C, Lee BC, Vandlen R, Keler T, Lauer P, Brockstedt D, Mellman I, Delamarre L: Antigen delivery to early endosomes eliminates the superiority of human blood BDCA3+ dendritic cells at cross presentation. J Exp Med 2013, 210:1049-1063.

41. Delneste Y, Magistrelli G, Gauchat J, Haeuw J, Aubry J, Nakamura K, KawakamiHonda N, Goetsch L, Sawamura T, Bonnefoy J, Jeannin P: Involvement of LOX-1 in dendritic cell-mediated antigen cross-presentation. Immunity 2002, 17:353-362.

42. Flamar AL, Xue Y, Zurawski SM, Montes M, King B, Sloan L, Oh S, Banchereau J, Levy Y, Zurawski G: Targeting concatenated HIV antigens to human CD40 expands a broad repertoire of multifunctional CD4+ and CD8+ T cells. Aids 2013, 27:2041-2051.

43. Tacken PJ, de Vries IJ, Gijzen K, Joosten B, Wu D, Rother RP, Faas SJ, Punt CJ, Torensma R, Adema GJ, Figdor CG: Effective induction of naive and recall T-cell responses by targeting antigen to human dendritic cells via a humanized anti-DC-SIGN antibody. Blood 2005, 106:1278-1285.

44. Seneschal J, Clark RA, Gehad A, Baecher-Allan CM, Kupper TS: Human epidermal Langerhans cells maintain immune homeostasis in skin by activating skin resident regulatory T cells. Immunity 2012, 36:873-884.

45. Kumamoto $Y$, Iwasaki A: Unique features of antiviral immune system of the vaginal mucosa. Curr Opin Immunol 2012, 24:411-416.

46. van der Aar AM, Picavet DI, Muller FJ, de Boer L, van Capel TM, Zaat SA, Bos JD, Janssen H, George TC, Kapsenberg ML, van Ham SM, Teunissen MB, de
Jong EC: Langerhans cells favor skin flora tolerance through limited presentation of bacterial antigens and induction of regulatory $\mathrm{T}$ cells. J Invest Dermatol 2013, 133:1240-1249.

47. Ma B, Forney LJ, Ravel J: Vaginal microbiome: rethinking health and disease. Annu Rev Microbiol 2012, 66:371-389.

48. Human Microbiome Project C: Structure, function and diversity of the healthy human microbiome. Nature 2012, 486:207-214.

49. Cash HL, Whitham CV, Behrendt CL, Hooper LV: Symbiotic bacteria direct expression of an intestinal bactericidal lectin. Science 2006, 313:1126-1130.

50. Mazmanian SK, Liu CH, Tzianabos AO, Kasper DL: An immunomodulatory molecule of symbiotic bacteria directs maturation of the host immune system. Cell 2005, 122:107-118.

51. Skinningsrud B, Lie BA, Husebye ES, Kvien TK, Forre O, Flato B, Stormyr A, Joner G, Njolstad PR, Egeland T, Undlien DE: A CLEC16A variant confers risk for juvenile idiopathic arthritis and anti-cyclic citrullinated peptide antibody negative rheumatoid arthritis. Ann Rheum Dis 2010, 69:1471-1474.

52. Zuvich RL, Bush WS, McCauley JL, Beecham AH, De Jager PL, International Multiple Sclerosis Genetics C, Ivinson AJ, Compston A, Hafler DA, Hauser SL, Sawcer SJ, Pericak-Vance MA, Barcellos LF, Mortlock DP, Haines JL: Interrogating the complex role of chromosome 16 p13.13 in multiple sclerosis susceptibility: independent genetic signals in the CIITA-CLEC16A-SOCS1 gene complex. Hum Mol Genet 2011, 20:3517-3524

53. Gene Expression Omnibus (GEO) [http://www.ncbi.nlm.nih.gov/geo]

doi:10.1186/s13073-014-0098-y

Cite this article as: Duluc et al.: Transcriptional fingerprints of antigen-presenting cell subsets in the human vaginal mucosa and skin reflect tissue-specific immune microenvironments. Genome Medicine 2014 6:98.

\section{Submit your next manuscript to BioMed Central and take full advantage of:}

- Convenient online submission

- Thorough peer review

- No space constraints or color figure charges

- Immediate publication on acceptance

- Inclusion in PubMed, CAS, Scopus and Google Scholar

- Research which is freely available for redistribution

Submit your manuscript at www.biomedcentral.com/submit
C Biomed Central 